\title{
Clustered Regularly Interspaced Short Palindromic Repeats (CRISPR): A Novel Genomic Modifying Technique
}

\author{
Ungkarit Wachapatthana, Tanat Thanupran
}

Mahidol University International Demonstration School, Thailand

Corresponding Author: Tanat Thanupran

\begin{abstract}
In a way akin to how technological advancements involving molecular and biological science rapidly developed during the last decade, CRISPR, a newly found genomic modifying technique, quickly gained the attention of the scientific community. This paper aims to provide a fundamental understanding of CRISPR technology by reviewing articles from several journals, consisting of general information about CRISPR technology, process, advantages, limitations, and comparisons with other technologies. This recent technology drastically altered the boundaries of genetic engineering-most notably due to its outstanding flexibility of gRNA modification, as demonstrated by the work of the two Nobel Prize award-winning scientists Emmanuelle Charpentier and Jennifer A. Doudna. From the journals that had been reviewed, this paper presents that the CRISPR-Cas9 is one of the most efficient tools for genetic engineering in our modern world because of its incredible potential to perform genetic material modification in a wide range of patients with greater efficiency, versatility, and accuracy than before, all the while being more cost-effective. Additionally, since significant research and newfound knowledge related to genetic science was made possible from the discovery of this CRISPR technology, the possibility of CRISPRCas9 treatment in patients-particularly to combat congenital genetic diseases-would become a horizon that is within the reach of humanity's hands.
\end{abstract}

Keywords: genomic modifying technique, CRISPR technology, genetic engineering, congenital genetic disease, genetic material modification

\section{INTRODUCTION}

All living things are said to be composed of tiny cells, the basic unit of life. The different types of cells in every organism are assigned specific instructions and roles that prolong life-sustaining processes-processes which, with their absence, would cause the very life within us to end (Martin, 2017).

Thus, a question would be: What determines such different roles of cells that compose not only our but all living organisms? The short answer is: All cells are composed of a certain particular genetic material called Deoxyribonucleic Acid, or abbreviated and known as DNA, which contains the "blueprint" to every activity that occurs within every organism (Watson et al., 1993).

DNAs are chains of nucleotides-a structure made from a 5-carbon sugar called deoxyribose, a nitrogenous base (of adenine, thymine, cytosine, and guanine), and a phosphate group-bonded in the form of a double helix (Arnott et al., 1974). Residing in the nucleoid of Prokaryotic cells and the nucleus of Eukaryotic cells, they code for proteins in a series of complex processes during transcription and translation upon receiving feedback from certain scenarios. In turn, such proteins make changes to the cell, respond to other conditions, and sometimes are sent to other cells. It is this 
different expression of "codes" from the DNA that determines a cell's activity, and thus its type (Davis et al., 1990). And as all cells compose our bodies, DNAs determine our characteristics and traits.

However, DNAs are said to be inherited-meaning it is passed onto an organism via their parents, can be mutated into other random forms, and do not only contain codes for beneficial traits but also some other inimical, detrimental effects (Benveniste et al., 1974). Hence, the main issue about DNA is that they can have unpredictable, undesirable coding patternsparticularly patterns which cause defects, grant an ineptness towards certain diseases, or culminate in inevitable forms of cancer and abnormalities (Srivastava et al., 1999).

However, all this can be avoided if humans could directly inspect, tweak and refine, and monitor the said genetic information that makes us, us (Handschuh et al., 1998). If this could be done in any way, it would mean the end for any unwanted characteristics, and perhaps even mean the removal of human "limiters" - the factor of what defines humanity's finite, restricted capabilities.

In the past, there were little to no techniques for editing genomes, and perhaps, it may not be an overstatement to say that scientists at that time had little knowledge about genetics (Nadeem et al., 2018). Nonetheless, as time passed, technology advancement dramatically developed and many discoveries of scientific techniques were made. In the genetic engineering and biotechnology aspects, a great scientific advancement would be the development of recombinant DNA technologies, which allows for a broad number of applications due to their ability to insert specific DNA segments into plasmids, and allowing the aforementioned DNA to express proteins and incorporate into mammalian (and likely, human) cells (Johnson, 1983). Therefore, it is not inconceivable that scientists would come up with a method for even more precise, accurate genetic modification; that is, they have come up with CRISPR.

A modern, powerful potential for gene editing, CRISPR (Clustered Regularly Interspaced Short Palindromic Repeats) is a cluster of DNA sequences derived from the fragments of DNA of bacteriophages appearing in the prokaryote's genomes (Ledford, 2015). Many said that there is a multitude of excitement that has been procreated by CRISPR in the field of science and technology. This is due to the fact of its accuracy, and efficiency which is more than other techniques of genome editing (Enriquez, 2021).

\section{Process}

To fully understand the key elements of the CRISPR-Cas9 genome editing method, it is crucial to be able to define each letter of CRISPR, as it may often cause confusion in what it is simply referring to.

Bacteria and Archaea organisms, akin to humans, have their own "immune system"; that is, despite belonging to another domain according to biological taxonomy, they have certain defense mechanisms against potential threats to their well-being (Weiss \& Schaible, 2015). And, it is one of these defense mechanisms which scientists have adapted as a method of treating diseases and interacting with specific genetic coding (Johnson \& Hug, 2019).

CRISPR-Cas9 is derived from the relationship between prokaryotic bacteria and bacteriophages (also commonly known as just "phages"), viruses that attack bacteria and many other prokaryotic organisms. Discovered by Frederick William Twort in 1915, and subsequently Felix d'Herelle, two years later in 1917, bacteriophages-directly translated as "bacteria eater" from the world "phagein" meaning "to eat"-are viruses (essentially genetic material surrounded by a protein structure) with high bactericidal abilities, capable of destroying prokaryotic organisms belonging to both Bacteria and Archaea domains (Twort, 1936). With the symbiotic 
and ubiquitous nature of organisms-both of those perceivable and imperceivable via the naked human eye-it is irrefutable that, like other members of nature, phages hold key roles in our current microbiological processdriven world.

Phages, diverse and abundant in the form of thousands of varieties, are all composed of three basic capsids (head) structural forms: as an icosahedral (meaning 20-sided) head with a tail known as a headtail phage, as an icosahedral without a tail simply known as an icosahedral phage, or found in a filamentous form as a filamentous phage (Rao \& Black, 2010). Bacteriophages can be classified roughly into two groups through the method the bacteriophage uses to replicate; that is, they are divided into virulent bacteriophages which replicate with the lytic cycle (or cytoplasmic viral replication) and temperate bacteriophages which replicate with the lysogenic cycle (Bertani, 1953). However, it is worth mentioning that some temperate bacteriophages are able to determine whether they should opt for the lytic cycle instead of the lysogenic cycle, depending on the current environment that they are present in.

Following a series of steps of a phage attaching to specific receptors of a bacterium and injecting its genetic materialbe it DNA or RNA, single-stranded (ss) or double-stranded (ds)-by means of a mechanism (contraction of the bacteriophage tail sheath) similar to a hypodermic needle, phages divert into the two different cycle replications (GuerreroFerreira et al., 2011).

Generally, in the lytic cycle, the injected viral genome circularizes and hijacks the cell and turns it into a "bacteriophage factory" where the cell's contents replicate, transcribe, and translate components of new progeny phages before the contents burst out of the cell in a process called lysis. (It is important to note that some lytic phages may postpone lysis when extracellular lytic phage concentrations are high. This is known as lysis inhibition.)
However, during lysogeny in lysogenic cycles, the injected viral genetic material either merges itself with the host bacterial cell's genetic material or remains separate as a plasmid, and the post-lysogeny genome is now known as a prophage. Following this occurrence, the resulting cell, now known as a lysogen, is allowed to survive as the prophage remains dormant or inactive, which, when undergoing cell division, can produce even more prophages (Little, 2005). This persists until induction; that is, the viral genome becomes activated, cuts itself out of the bacterial chromosome, and re-enters the lytic cycle where it replicates more bacteriophages before lysing out of the host cell, ensuing ideal conditions or external environmental cues such as low extracellular phage concentrations, or host cell deterioration or malnutrition (Bratbak et al., 1998).

However, as nature developed, so did the host organisms; in other words, they began to evolve different types of protective mechanisms to render themselves immune to the dangerous, parasitic nature of bacteriophages. Likewise, one of those defense mechanisms that had been observed in sample organisms such as Escherichia coli. was CRISPR (Diez-Villasenor, 2010). This mechanism is capable of identifying foreign nucleic material and destroying it, which, therefore, prevents the destructive repercussions ensuing the embedding of said foreign DNA or RNA from bacteriophages into the host bacterium's genome.

CRISPR-Cas9 derives from the CRISPR locus-composed of a cluster of regularly interspaced, alternating pattern of short palindromic repeats (hence the name CRISPR), usually of around 23-47 base pairs, and spacer (or protospacer) nucleotide sequences, 21-72 bp long-and its work in conjunction with cas genes (CRISPR associated proteins). Spacer DNA sequences function as copies of bacteriophage DNA, which can be transcribed by RNA polymerases into CRISPR RNA (precrRNAs) and later divided by specific 
endoribonucleases (ribonucleases which cleave RNA internally) into smaller, functional CRISPR RNAs (crRNAs) that are partial complementary base pairs to the invading foreign DNA (Grosschedl \& Birnstiel, 1980). These crRNAs bind with another RNA molecule known as transactivating crRNA (tracrRNA), transcribed from genes at a region following the CRISPR and spacer sequences, forming a guide RNA (gRNA) (Brendel, 2014). On the other hand, the cas genes can be transcribed and translated into cas protein complexes which serve as endonucleases, or enzymes that can cleave the invading genetic material. With the two components combined, the gRNA acts as a foreign genetic material identifier for the cas complex, as when the two comes in contact with a particular DNA sequence, if the gRNA matches perfectly with a part of the nucleotide sequence, the complex snips off the invading nucleic acid into two and renders the gene as deactivated (Gasiunas et al., 2012). The viral genetic sequence, now cut into two, either remains deactivated or, if the cell tries to repair it, forms random mutations which deflect the dangerous abilities or instruction of the viral DNA and render it ineffective, with the former being the general case.

However, a question would be: How would the viral DNA be dealt with if there were no previous records or spacer sequences which match with a particular, new invading phage? This can be explained that the cas genetic sequence also codes for another type of cas protein (generally class 1 cas proteins) which allows for the viral DNA to be broken apart and made into a spacer sequence in the system (Canver et al., 2018). With this system, the seemingly inevitable fate of cell lysis is not only prevented but a part of the viral DNA is also recorded as part of the CRISPR locus in the form of a new spacer sequence so that future invasions can be dealt with in the same manner. As the bacterium reproduces and divides, these CRISPR and spacer sequences are inherited from parent bacteria as well (Deng et al., 2012). Simply put, as an analogy, CRISPR-Cas systems are similar to our human immune system, with leukocytes and immunoglobulins, but on a smaller scale (Thielen et al., 2018).

Following this knowledge and understanding of the CRISPR-Cas defense mechanism of bacteria and archaea, the two Nobel Prize in Chemistry award-winning scientists Emmanuelle Charpentier and Jennifer A. Doudna, together with several other individuals, figured a way of making good use out of the system-they figured that they can modify the gRNA of the protein complex (Kiani et al., 2015). With the gRNA's role in the mechanism being the "identifier" of who and where to cut, humans can synthesize our own specific line of crRNA and match it with a corresponding tracrRNA (Chylinski et al., 2013). Consequently, the so-called "genetic scissor" can now serve any need of gene modification, as any identified genetic sequence may be cut, and later inserted with any specific sections of nucleotide sequencing per the scientists' needs (Petersen \& Niemann, 2015).

However, it is important to note that the aforementioned knowledge only entails a general part of CRISPR-based genetic engineering. In reality, there are many variations of cas9 that provide different advantages and disadvantages, guide RNAs may even be divided into other formats including single guide RNAs (sgRNAs) which contain a connected crRNA and tracrRNA piece, or into two-piece guide RNAs known as CRISPR tracer RNAs (crtracrTNAs), and so on (Jiang et al., 2015). Moreover, there is much more information that allows for specific exceptions and circumstances. Thus, there are many topics regarding CRISPR genetic manipulation that need to be reviewed so as to avoid controversial issues that may occur due to low editing efficacy.

\section{Advantages}

As technological advancement has developed over time, new techniques for 
altering genes-CRISPR has ultimately been introduced. In the past, techniques for gene editing were quite intricate and overpriced; in 1987, Yoshizumi Ishino, a Japanese molecular biologist, along with his colleagues, discovered the CRISPR loci (Ishino et al., 2018). Many at that time were enthusiastic about the discovery of CRISPR because the potential that lies within the system came up with many preferences that helped scientists perform genome editing better. Focusing on its simplicity and efficiency, this technique was tremendously simpler, cheaper, and easier to use compared with other previous techniques in the past (Lino et al., 2018). To illustrate, CRISPR-Cas9 is a technique used to find a target bit of DNA that is located inside a cell and change the specific pieces of target DNA; while other techniques had such complicated processes (Dance, 2015).

Not only its simplicity and efficiency made it widely used, but also it had such a potentially unique function to cure disease. (Lundberg \& Novak, 2015) The discover of this recent technology helped extend genetic research and experiments in the modern world and border on developed gene therapy which involved the importance of the advancement of the developed technology for curing a multitude of genetic diseases. Whereas the previously traditional gene therapy had suffered with its reliance as it was able to cause mutation and damage from chemical toxin, which were insertional oncogenesis and immunogenic toxicity, respectively; CRISPR-Cas9, as it is simpler, faster and more efficient, can be easily used as disease therapy by just knocking out a non-working or missing gene and replacing it with another gene (Koo et al., 2017). This process can be done by packaging a working gene in a transport vehicle which is known as a vector. Its task is to bring a particular gene into the specific cells' nucleus. When it arrives, it may either be enhanced to be one part of the DNA of that specific cell or stay unconnected. Moreover, that vector also has to choose either make the missing protein or provide that protein in short. Once no longer used, that vector is ultimately eradicated (Xu et al., 2019).

Besides other applications, as this recent genome editing technique could directly apply to the embryo, time spent for modifying specifically target genes is much less than the genome targeting techniques based upon the use of embryonic stem cells (Morrison \& de Saille, 2019). From the deduction of time which allows scientists to rapidly create cells and the models of animals. Also, the researchers will definitely have more time to research for the genetic infectious diseases; including cancer, prion disease, malaria, to name a few (Khan et al., 2016). Therefore, the CRISPR-Cas9 technique has dramatically developed the technological advancement of genome editing techniques which could also involve the issue of scientific uses.

\section{Limitations}

Following the basic understanding of how CRISPR-Cas9 systems work, we begin after the successful splintering of the foreign genetic material into two. As aforementioned, oftentimes the cell would attempt to repair the DNA to its former. During this process of cell DNA repair and restoration, there are two well-known pathways: Non-Homologous End-Joining (NHEJ) or Homology-Directed Repair (HDR) (Miyaoka et al., 2016).

NHEJ simply means that the DNA repair occurs randomly; that is, with normal genetic material mutations such as insertion and deletion (Burma et al., 2006). In this default case, it is true that, since its nature works as an error-prone mechanism where the DNA is not restored to its original form but to a form that does not function as before, the NHEJ is cost-effective and most of the time useful for therapeutic scenarios (Caracciolo et al., 2020). However, because of the same error-prone mechanism, there is a small chance of the process malfunctioning and creating even more concerns for the cell (Englund, 2017). 
As for HDR, it means that a template is required to repair the DNA-CRISPR technology can be used to provide another homologous pair of DNA that, when the damaged DNA lines up with, allows for uncanny replication repair via homologous recombination (Zhang et al., 2017). This method allows for more accurate editing, and thus would be preferred over the NHEJ. Although this is true for the HDR, unfortunately, as of current, the precise genetic editing that comes with HDR is highly inefficient. This is because of the strict condition that the template that contains homology arms must correspond to the separated DNA's base pairs (Yao et al., 2017). As a consequence, NHEJ, though less accurate, is often seen as the better fit for clinical therapeutic uses. This is also paired with other minor reasons; for instance, the NHEJ can take place during the G1, S, and G2 phases of the cell cycle, whereas HDR is found to be predominant in only late $\mathrm{S}$ and $\mathrm{G} 2$ phases (i.e. the NHEJ allows for more flexibility of time period) (Romero et al., 2019).

Another drawback of the CRISPRCas9 system is that some variations of it require the usage of a protospacer adjacent motif (PAM). As part of the invading DNA sequence, PAM allows for the differentiation between self and foreign genetic material (Karvelis et al., 2015). The Cas9 protein cannot cleave any genetic material that does not have an adjacent PAM, and moreover, to stress the importance of PAM, it is critical to understand that it is also this PAM sequence that gRNAs use to recognize foreign invaders. Therefore, because there are many variants of PAM sequences that are unique to different bacteria and since PAM may be oftentimes unavailable in the desired gene loci, genetic engineers must devise a system in which Cas9 proteins correspond to different PAM sequences so that genetic engineering can take place with fewer constraints (Zhao et al., 2017). Otherwise, another option is to create a Cas9 version that does not involve the existence of a
PAM sequence, allowing for a similar result of being able to target more DNAs.

Other than the complications stated above, there are other minor common problems that arise as well. This includes:

- Immunotoxicity: In some cases, the body of the patient contains anti-Cas 9 immunogenic responses, or some processes may trigger innate immunities that do not accept Cas9 proteins, and thus, render CRISPR-Cas9 clinical therapies ineffective and dangerous (Uddin et al., 2020).

- Ethical issues: Due to the fact that CRISPR-Cas9 systems are genetic editing methods, the possibility of human germline editing-engineering genetic materials of gametes for the purpose of disease prevention and trait enhancement-still remains controversial to many (Mulvihill et al., 2017). Thus, until an international framework has been established over all aspects, scientists have proposed an indefinite global moratorium on genetically modified children, following the first CRISPR germline editing implant in human embryos that had occurred in 2017 and the concerns that had ensued after.

- Off-target Effects: The utilization of different Cas9 variants often cause offtarget effects (OTEs)-effects that may cause random mutations and genomic instability-to manifest and disrupt the normally functional genes (Cho et al., 2014). Consequently, Cas9 variants must be fully understood to such an extent that most off-target activities are reduced to a zero.

\section{Comparison with other technologies}

Before the technological development had been advanced and led to the discoveration of CRISPR-Cas9, there were other previous techniques for genome editing that were once authorized to modify and express the targeted genomic sequence also. The aforementioned techniques included zinc finger nucleases (ZFNs), and 
transcription activator-like effector nucleases (TALENs) (Gaj et al., 2013).

The oldest engineered DNA-binding protein, ZFNs, is one technique for genome editing involving the breaking of a doublestrand of DNA at specific positions. This is significant in that it is considered as sitespecific mutagenesis, which helps stimulate the DNA-repair process (De Pater et al., 2009). Another well-known technique for curing specific DNA sequences, TALENs are restriction enzymes that can be found in plant-pathogenic bacteria; specifically in the genus Xanthomonas, which is used when an infection is occurred in a plant. It can be used to target the specific genomic sequence by a recognition of a difference in a single DNA base's combination (Joung \& Sander, 2013).

Comparing those previous aforementioned techniques with CRISPRCas9, each of them have their own unique pros and cons. Focusing on simplicity and efficiency, CRISPR noticeably performs well with easier processes and higher efficiency meaning that it functions better at targeting genome sequences compared to other techniques. For the requirements being used, CRISPR-Cas9 only requires simple 20nt change, whereas both ZFNs and TALENs need two large proteins (Zhang et al., 2019). On top of that, while the other two systems are both man-made, CRISPRCas9 is derived from prokaryotic organisms known as bacteria. Naturally, this system starts itself to function when a foreign pathogen or virus enters a bacterium. With Cas9 proteins, the system is able to cut off the viral DNA part and initially assemble that part to CRISPR locus of the particular genome of bacteria such that the viral DNA will be inactive by the binding of Cas proteins to the target when the same virus invades that bacterium again (Boettcher \& McManus, 2015). All in all, it is undeniable that CRISPR-Cas9 is simpler, cheaper, higher efficiency and also can be used in various ways compared to those previous systems of genomic editing which reveals the development in both scientific and technological advancements.

\section{Acknowledgement: None}

\section{Conflict of Interest: None}

\section{Source of Funding: None}

\section{REFERENCES}

1. Arnott, S., Chandrasekaran, R., Hukins, D. W. L., Smith, P. J. C., \& Watts, L. (1974). Structural details of a double-helix observed for DNAs containing alternating purine and pyrimidine sequences. Journal of molecular biology, 88(2), 523-533.

2. Benveniste, R. E., \& Todaro, G. J. (1974). Evolution of C-type viral genes: inheritance of exogenously acquired viral genes. Nature, 252(5483), 456-459.

3. Bertani, G. (1953, January). Lysogenic versus lytic cycle of phage multiplication. In Cold Spring Harbor symposia on quantitative biology (Vol. 18, pp. 65-70). Cold Spring Harbor Laboratory Press.

4. Boettcher, M., \& McManus, M. T. (2015). Choosing the right tool for the job: RNAi, TALEN, or CRISPR. Molecular cell, 58(4), 575-585.

5. Bratbak, G., Jacobsen, A., Heldal, M., Nagasaki, K., \& Thingstad, F. (1998). Virus production in Phaeocystis pouchetii and its relation to host cell growth and nutrition. Aquatic Microbial Ecology, 16(1), 1-9.

6. Brendel, J., Stoll, B., Lange, S. J., Sharma, K., Lenz, C., Stachler, A. E., ... \& Marchfelder, A. (2014). A complex of Cas proteins 5,6 , and 7 is required for the biogenesis and stability of clustered regularly interspaced short palindromic repeats (crispr)-derived rnas (crrnas) in Haloferax volcanii. Journal of Biological Chemistry, 289(10), 7164-7177.

7. Burma, S., Chen, B. P., \& Chen, D. J. (2006). Role of non-homologous end joining (NHEJ) in maintaining genomic integrity. DNA repair, 5(9-10), 1042-1048.

8. Canver, M. C., Joung, J. K., \& Pinello, L. (2018). Impact of genetic variation on CRISPR-Cas targeting. The CRISPR journal, 1(2), 159-170.

9. Caracciolo, D., Riillo, C., Arbitrio, M., Di Martino, M. T., Tagliaferri, P., \& Tassone, P. (2020). Error- prone DNA repair 
pathways as determinants of immunotherapy activity: an emerging scenario for cancer treatment. International journal of cancer, 147(10), 2658-2668.

10. Cho, S. W., Kim, S., Kim, Y., Kweon, J., Kim, H. S., Bae, S., \& Kim, J. S. (2014). Analysis of off-target effects of CRISPR/Cas-derived RNA-guided endonucleases and nickases. Genome research, 24(1), 132-141.

11. Chylinski, K., Le Rhun, A., \& Charpentier, E. (2013). The tracrRNA and Cas9 families of type II CRISPR-Cas immunity systems. RNA biology, 10(5), 726-737.

12. Dance, A. (2015). Core Concept: CRISPR gene editing. Proceedings of the National Academy of Sciences, 112(20), 6245-6246.

13. Davis, R. L., Cheng, P. F., Lassar, A. B., \& Weintraub, H. (1990). The MyoD DNA binding domain contains a recognition code for muscle-specific gene activation. Cell, 60(5), 733-746.

14. De Pater, S., Neuteboom, L. W., Pinas, J. E., Hooykaas, P. J., \& Van Der Zaal, B. J. (2009). ZFN-induced mutagenesis and gene-targeting in Arabidopsis through Agrobacterium-mediated floral dip transformation. Plant biotechnology journal, 7(8), 821-835.

15. Deng, L., Kenchappa, C. S., Peng, X., She, Q., \& Garrett, R. A. (2012). Modulation of CRISPR locus transcription by the repeatbinding protein $\mathrm{Cbp} 1$ in Sulfolobus. Nucleic acids research, 40(6), 2470-2480.

16. Díez-Villaseñor, C., Almendros, C., GarcíaMartínez, J., \& Mojica, F. J. (2010). Diversity of CRISPR loci in Escherichia coli. Microbiology, 156(5), 1351-1361.

17. Englund, B. (2017). Rabbit Polyclonal Antibodies to Detect C7orf49 Proteins Toward Understanding Double Strand Break Repair. The FASEB Journal, 31, 9065.

18. Enríquez, P. (2021). Rewriting Nature: The Future of Genome Editing and how to Bridge the Gap Between Law and Science. Cambridge University Press.

19. Gaj, T., Gersbach, C. A., \& Barbas III, C. F. (2013). ZFN, TALEN, and CRISPR/Casbased methods for genome engineering. Trends in biotechnology, 31(7), 397-405.

20. Gasiunas, G., Barrangou, R., Horvath, P., \& Siksnys, V. (2012). Cas9-crRNA ribonucleoprotein complex mediates specific DNA cleavage for adaptive immunity in bacteria. Proceedings of the National Academy of Sciences, 109(39), E2579-E2586.

21. Grosschedl, R., \& Birnstiel, M. L. (1980). Spacer DNA sequences upstream of the TATAAATA sequence are essential for promotion of $\mathrm{H} 2 \mathrm{~A}$ histone gene transcription in vivo. Proceedings of the National Academy of Sciences, 77(12), 7102-7106.

22. Guerrero-Ferreira, R. C., Viollier, P. H., Ely, B., Poindexter, J. S., Georgieva, M., Jensen, G. J., \& Wright, E. R. (2011). Alternative mechanism for bacteriophage adsorption to the motile bacterium Caulobacter crescentus. Proceedings of the National Academy of Sciences, 108(24), 9963-9968.

23. Handschuh, S., Wagener, M., \& Gasteiger, J. (1998). Superposition of threedimensional chemical structures allowing for conformational flexibility by a hybrid method. Journal of Chemical Information and Computer Sciences, 38(2), 220-232.

24. Ishino, Y., Krupovic, M., \& Forterre, P. (2018). History of CRISPR-Cas from encounter with a mysterious repeated sequence to genome editing technology. Journal of bacteriology, 200(7), e00580-17.

25. Jiang, F., Zhou, K., Ma, L., Gressel, S., \& Doudna, J. A. (2015). A Cas9-guide RNA complex preorganized for target DNA recognition. Science, 348(6242), 1477-1481.

26. Johnson, I. S. (1983). Human insulin from recombinant DNA technology. Science, 219(4585), 632-637.

27. Johnson, L. A., \& Hug, L. A. (2019). Distribution of reactive oxygen species defense mechanisms across domain bacteria. Free Radical Biology and Medicine, 140, 93-102.

28. Joung, J. K., \& Sander, J. D. (2013). TALENs: a widely applicable technology for targeted genome editing. Nature reviews Molecular cell biology, 14(1), 49-55.

29. Karvelis, T., Gasiunas, G., Young, J., Bigelyte, G., Silanskas, A., Cigan, M., \& Siksnys, V. (2015). Rapid characterization of CRISPR-Cas9 protospacer adjacent motif sequence elements. Genome biology, 16(1), 1-13.

30. Khan, F. A., Pandupuspitasari, N. S., ChunJie, H., Ao, Z., Jamal, M., Zohaib, A., ... \& ShuJun, Z. (2016). CRISPR/Cas9 
therapeutics: a cure for cancer and other genetic diseases. Oncotarget, 7(32), 52541.

31. Kiani, S., Chavez, A., Tuttle, M., Hall, R. N., Chari, R., Ter-Ovanesyan, D., ... \& Church, G. (2015). Cas9 gRNA engineering for genome editing, activation and repression. Nature methods, 12(11), 10511054.

32. Koo, T., Yoon, A. R., Cho, H. Y., Bae, S., Yun, C. O., \& Kim, J. S. (2017). Selective disruption of an oncogenic mutant allele by CRISPR/Cas9 induces efficient tumor regression. Nucleic acids research, 45(13), 7897-7908.

33. Ledford, H. (2015). CRISPR, the disruptor. Nature News, 522(7554), 20.

34. Lino, C. A., Harper, J. C., Carney, J. P., \& Timlin, J. A. (2018). Delivering CRISPR: a review of the challenges and approaches. Drug delivery, 25(1), 1234-1257.

35. Little, J. W. (2005). Lysogeny, prophage induction, and lysogenic conversion. Phages: their role in bacterial pathogenesis and biotechnology, 37-54.

36. Lundberg, A. S., \& Novak, R. (2015). CRISPR-Cas gene editing to cure serious diseases: treat the patient, not the germ line. The American Journal of Bioethics, 15(12), 38-40.

37. Martin, R. (2017). Basic structures of prokaryotic and eukaryotic cells. Microreviews in Cell and Molecular Biology, 2(2).

38. Miyaoka, Y., Berman, J. R., Cooper, S. B., Mayerl, S. J., Chan, A. H., Zhang, B., ... \& Conklin, B. R. (2016). Systematic quantification of HDR and NHEJ reveals effects of locus, nuclease, and cell type on genome-editing. Scientific reports, 6(1), 112.

39. Morrison, M., \& de Saille, S. (2019). CRISPR in context: towards a socially responsible debate on embryo editing. Palgrave Communications, 5(1), 1-9.

40. Mulvihill, J. J., Capps, B., Joly, Y., Lysaght, T., Zwart, H. A., \& Chadwick, R. (2017). Ethical issues of CRISPR technology and gene editing through the lens of solidarity. British medical bulletin, 122(1), 17-29.

41. Nadeem, M. A., Nawaz, M. A., Shahid, M. Q., Doğan, Y., Comertpay, G., Yıldız, M., ... \& Baloch, F. S. (2018). DNA molecular markers in plant breeding: current status and recent advancements in genomic selection and genome editing. Biotechnology \&
Biotechnological Equipment, 32(2), 261285.

42. Petersen, B., \& Niemann, H. (2015). Molecular scissors and their application in genetically modified farm animals. Transgenic research, 24(3), 381-396.

43. Rao, V. B., \& Black, L. W. (2010). Structure and assembly of bacteriophage T4 head. Virology journal, 7(1), 1-14.

44. Romero, Z., Lomova, A., Said, S., Miggelbrink, A., Kuo, C. Y., CampoFernandez, B., ... \& Kohn, D. B. (2019). Editing the sickle cell disease mutation in human hematopoietic stem cells: comparison of endonucleases and homologous donor templates. Molecular Therapy, 27(8), 1389-1406.

45. Srivastava, V., Anderson, O. D., \& Ow, D. W. (1999). Single-copy transgenic wheat generated through the resolution of complex integration patterns. Proceedings of the National Academy of Sciences, 96(20), 11117-11121.

46. Thielen, A. J., van Baarsen, I. M., Jongsma, M. L., Zeerleder, S., Spaapen, R. M., \& Wouters, D. (2018). CRISPR/Cas9 generated human CD46, CD55 and CD59 knockout cell lines as a tool for complement research. Journal of immunological methods, 456, 15-22.

47. Twort, F. W. (1936). Further investigations on the nature of ultra-microscopic viruses and their cultivation. Epidemiology \& Infection, 36(2), 204-235.

48. Uddin, F., Rudin, C. M., \& Sen, T. (2020). CRISPR gene therapy: applications, limitations, and implications for the future. Frontiers in Oncology, 10, 1387.

49. Watson, J. D., \& Crick, F. H. (1993). Genetical implications of the structure of deoxyribonucleic acid. JAMA, 269(15), 1967-1969.

50. Weiss, G., \& Schaible, U. E. (2015). Macrophage defense mechanisms against intracellular bacteria. Immunological reviews, 264(1), 182-203.

51. Xu, X., Wan, T., Xin, H., Li, D., Pan, H., Wu, J., \& Ping, Y. (2019). Delivery of CRISPR/Cas9 for therapeutic genome editing. The journal of gene medicine, 21(7), e3107.

52. Yao, X., Wang, X., Liu, J., Hu, X., Shi, L., Shen, X., ... \& Yang, H. (2017). CRISPR/Cas9-Mediated precise targeted integration in vivo using a double cut donor 
Ungkarit Wachapatthana et.al. Clustered Regularly Interspaced Short Palindromic Repeats (CRISPR): a novel genomic modifying technique

with short homology arms. EBioMedicine, 20, 19-26.

53. Zhang, J. P., Li, X. L., Li, G. H., Chen, W., Arakaki, C., Botimer, G. D., ... \& Zhang, X. B. (2017). Efficient precise knockin with a double cut HDR donor after CRISPR/Cas9mediated double-stranded DNA cleavage. Genome biology, 18(1), 1-18.

54. Zhang, J., Liu, J., Yang, W., Cui, M., Dai, B., Dong, Y.,... \& Cang, M. (2019). Comparison of gene editing efficiencies of CRISPR/Cas9 and TALEN for generation of MSTN knock-out cashmere goats. Theriogenology, 132, 1-11.
55. Zhao, C., Zheng, X., Qu, W., Li, G., Li, X., Miao, Y. L.,... \& Zhao, S. (2017). CRISPRoffinder: a CRISPR guide RNA design and off-target searching tool for user-defined protospacer adjacent motif. International journal of biological sciences, 13(12), 1470.

How to cite this article: Wachapatthana $U$, Thanupran T. Clustered Regularly Interspaced Short Palindromic Repeats (CRISPR): a novel genomic modifying technique. International Journal of Science \& Healthcare Research. 2021; 6(3): 254-263. DOI: https://doi.org/ 10.52403/ijshr.20210744 